\title{
Identification and Management of Immune Checkpoint Inhibitor-Related Myocarditis: Use Troponin Wisely
}

\author{
Paolo Spallarossa, MD¹; Giacomo Tini, MD ${ }^{1,2}$; Matteo Sarocchi, MD ${ }^{1,2}$; Eleonora Arboscello, MD ${ }^{1}$; Francesco Grossi, MD³ \\ Paola Queirolo, MD ${ }^{1}$; Gabriele Zoppoli, MD, PhD ${ }^{1,2}$; and Pietro Ameri, MD, PhD ${ }^{1,2}$
}

\section{Background}

Myocarditis is an uncommon, but fearsome complication of immune checkpoint inhibitors (ICPis) because it may result in life-threatening heart failure, cardiogenic shock, and arrhythmias. ${ }^{1-4}$ Among more than 20,000 patients treated with nivolumab and/or ipilimumab until April 2016 and included in a safety database of Bristol-Myers Squibb regardless of adverse events (AEs), 0.09\% experienced myocarditis and less than $0.03 \%$ experienced fatal myocarditis. ${ }^{2}$ Of all 964 patients at the Massachusetts General Hospital who received ICPis between November 2013 and July 2017, 1.14\% developed myocarditis and $0.52 \%$ had major cardiovascular AEs. ${ }^{3}$ According to a recent study, 122 cases of ICPi-related myocarditis were recorded since 2013 in VigiBase, the WHO database of individual patient safety reports. ${ }^{4}$ Classification was severe in $84 \%$, and death was more frequent with combination than with single immune therapy $(66 \% \vee 40 \%)$.

The analysis of VigiBase confirmed that myocarditis is strongly associated with ICPis because reports of this AE between 2008 and 2018 were 11-fold higher for ICPis than for all drugs. ${ }^{4}$ Furthermore, the analysis showed that the prevalence of myocarditis after ICPis has steadily increased over the years, which suggests that the burden of this complication is growing. This trend may be ascribed to various factors and is predicted to become even stronger in the near future. First, ICPis are being prescribed to a progressively larger population, which increases the absolute number of individuals who are expected to have myocarditis, even if the percent of the total treated patients remains low. ${ }^{5}$ Moreover, it is conceivable that myocarditis was missed in a nonnegligible number of patients during the initial experience with ICPis because myocarditis often has an insidious clinical presentation or a relatively benign, indolent course and, therefore, may not be recognized. Now, it is known that ICPis can cause myocarditis, and more attention is being paid to this AE than previously; thus, atypical or smoldering forms of the disease also are likely to be diagnosed. ${ }^{4}$
On the basis of these data, active screening for myocarditis of patients who receive ICPis has been proposed by measuring cardiac troponin (cTn), especially during the first weeks of treatment when most cases of ICPi-associated myocarditis seem to occur. ${ }^{2,6}$ This recommendation aims at further improving the early detection of myocarditis and prompting its appropriate management to forestall a potentially lethal evolution. Nonetheless, it also promotes extensive cTn testing, which may not be sustainable unless some concepts, well known by the cardiologist but not necessarily familiar to the oncologist, are kept in mind.

\section{What cTn Measures and What Elevated Levels Mean}

Both isoforms of cTn, cTnl and cTnT, are components of the contractile apparatus of cardiomyocytes. When cardiomyocytes are damaged, they are released into the extracellular space and eventually into the bloodstream. ${ }^{7}$ Noncardiac tissues do not normally express cTn, which is thereby cardiac specific. Nevertheless, a number of conditions that affect the heart lead to cTn concentrations above the upper reference limit (URL). ${ }^{7}$

Among cardiac diseases that underlie a rise in cTn, acute coronary syndrome (ACS) is the most frequent. It may be the consequence of sudden coronary artery obstruction, typically by a thrombus that has grown at the surface of an atherosclerotic plaque. ${ }^{8}$ ACS also may result from an imbalance between myocardial oxygen demand and blood supply, with the latter not increasing proportionally with the former; this most often happens because of preexisting coronary lesions that have narrowed the artery lumen and limit blood flow. ${ }^{8}$ Noncardiac disorders, such as chronic kidney disease or pneumonia, also may directly or indirectly elicit myocardial injury. In fact, the cause of increased cTn levels in hospitalized patients is most frequently noncardiac or multifactorial. ${ }^{9}$

Germane to the surveillance of patients who receive ICPis, cTnT may no longer be cardiac specific in the presence of skeletal muscle disorders. In this context, elevated concentrations of CTnT were demonstrated 
despite normal heart structure and function possibly because commercial assays detect $T n$ isoforms expressed by the diseased muscle. ${ }^{10}$ Because myositis is an emerging immune-related AE of ICPis, ${ }^{11}$ this possibility should not be overlooked. Likewise, mild elevations in CTn may be the result of inflammation of the subepicardial myocardium during pericarditis. ${ }^{4}$ This is important in view of the evidence that pericarditis is another immune-related $A E$ of ICPis. ${ }^{4}$ Finally, circulating $\mathrm{CTn}$ is measurable in a substantial proportion of healthy individuals by current highsensitivity assays, ${ }^{13}$ with this indeed being the reason why the cutoff for abnormal values is set to above the 99th percentile of the URL.

With this background, cTn testing will help to diagnosis and support therapeutic choices only if it is related to the clinical context. Assessment of cTn levels regardless of the clinical scenario rarely provides clear-cut indications for changing and improving patient management. ${ }^{7}$ Conversely, cTn measurement may give prognostic information irrespective of the clinical situation because increased $\mathrm{cTn}$ values are associated with worse outcomes and higher mortality in the general population ${ }^{14}$ and in patients with both cardiac and noncardiac conditions. ${ }^{7,9}$

\section{Troponin Testing for ICPi-Associated Myocarditis}

The aforementioned considerations hold true for ICPi-related myocarditis. In general, endomyocardial biopsy is the gold standard to diagnose myocarditis. ${ }^{15}$ Being an invasive procedure, however, it is performed only when indicated by a work-up that includes clinical examination, ECG, biochemical markers of myocardial injury as well of inflammation, echocardiography, and cardiac magnetic resonance. ${ }^{15}$ This latter imaging technique is now capable of revealing myocardial inflammation with high accuracy to the point that endomyocardial biopsy often becomes unnecessary to reach the diagnosis of myocarditis and begin therapy. ${ }^{12}$ Thus, comprehensive evaluation of a patient with suspected ICPitriggered myocarditis is of utmost importance.

Alarming symptoms and signs, such as new-onset or worsening fatigue, chest pain, dyspnea, palpitation, peripheral edema, or hypotension, should be noted immediately and prompt an accurate clinical evaluation. ${ }^{5} \mathrm{~A}$ definite diagnosis of myocarditis relies on consistent symptoms and signs together with one or more of the following: newly abnormal ECG, cTn elevation, or new and otherwise unexplained functional and structural alterations on cardiac imaging. ${ }^{15}$ Consultation with a cardiologist is fundamental. ${ }^{16,17}$ However, the results of ECG and CTn testing may precede the cardiologist's evaluation and have to be interpreted by the physician who is administering the $\mathrm{ICPi}$, at least preliminarily. Any significant modification of the ECG should prompt the suspicion of an ongoing cardiac (or pericardial) disease. As far as cTn is concerned, we propose the following guiding points. First, an increase in cTn may indicate not only myocarditis but also myocardial injury as a result of other causes. Second, in particular, the possibility of ACS, which is far more common than myocarditis, must be taken into account; mildly elevated cTn also may be a feature of pericarditis. Third, ACS especially should be considered in cases of major cardiovascular risk factors, family history of ischemic heart disease at a young age, typical chest pain, and ECG changes limited to some leads that explore a definite region of the left ventricle. Fourth, a first abnormal cTn concentration must be followed by retesting (after 2 to 3 hours if a high-sensitivity assay is used) to determine the dynamics of cTn elevation; ACS is characterized by a typical rising and/or falling pattern of cTn values ( $v$ persistently increased values in myocarditis). ${ }^{8}$ Finally, cTn levels may be normal initially in myocarditis $^{18}$; if the clinical suspicion is high, ECG, cTn, and echocardiography should be repeated after 1 or a few days and be vigilant meanwhile.

In summary, measurement of cTn upon the appearance of suggestive cardiac symptoms and signs is appropriate and consistent with the state-of-the-art approach to any myocarditis (Fig 1A). The only caveat is to read the results of the test correctly. The level of alarm must be high in the suspicion of myocarditis, and direct referral to an emergency department may be appropriate.

By contrast, the issue of whether cTn should be routinely assessed in asymptomatic patients receiving ICPis is unresolved. ASCO suggests consideration of baseline cTn determinations, especially in patients who are receiving combination immune therapy, because a basal value of cTn may be helpful in cases of subsequent unclear symptoms or equivocal diagnostic examinations. ${ }^{16}$ No indication is provided about serial cTn measurements because of the lack of supporting evidence. Similarly, the Toxicity Management Working Group of the Society for Immunotherapy of Cancer recommends CTn assessment at baseline while highlighting the uncertainty about the cost effectiveness of repeated cTn testing given the rarity of ICPi-associated myocarditis and the paucity of available data. ${ }^{17}$ Of note, the working group also states that an accurate cardiovascular evaluation should be done before initiating ICPis, with participation of a cardiologist if needed. ${ }^{17}$ This pretreatment visit is invaluable because it provides information that may allow for better management of subsequent cardiovascular events. For example, estimation of the likelihood of ACS will be more accurate if the cardiovascular risk factor profile is known. Moreover, having a basal ECG is prerequisite for analyzing any change during therapy.

Some expert centers systematically measure cTn at regular intervals during the first weeks of combination immunotherapy. 2,6 This strategy is motivated by the fact that myocarditis is more frequent and severe with dual than with single ICPi treatment and may be fatal; thus, it must be looked for irrespectively of the clinical scenario. It is also based on the assumption that most of the time, an increase in CTn during ICPi therapy corresponds to some degree of myocarditis, as it does the elevation in cTn that accompanies typical symptoms, evident 


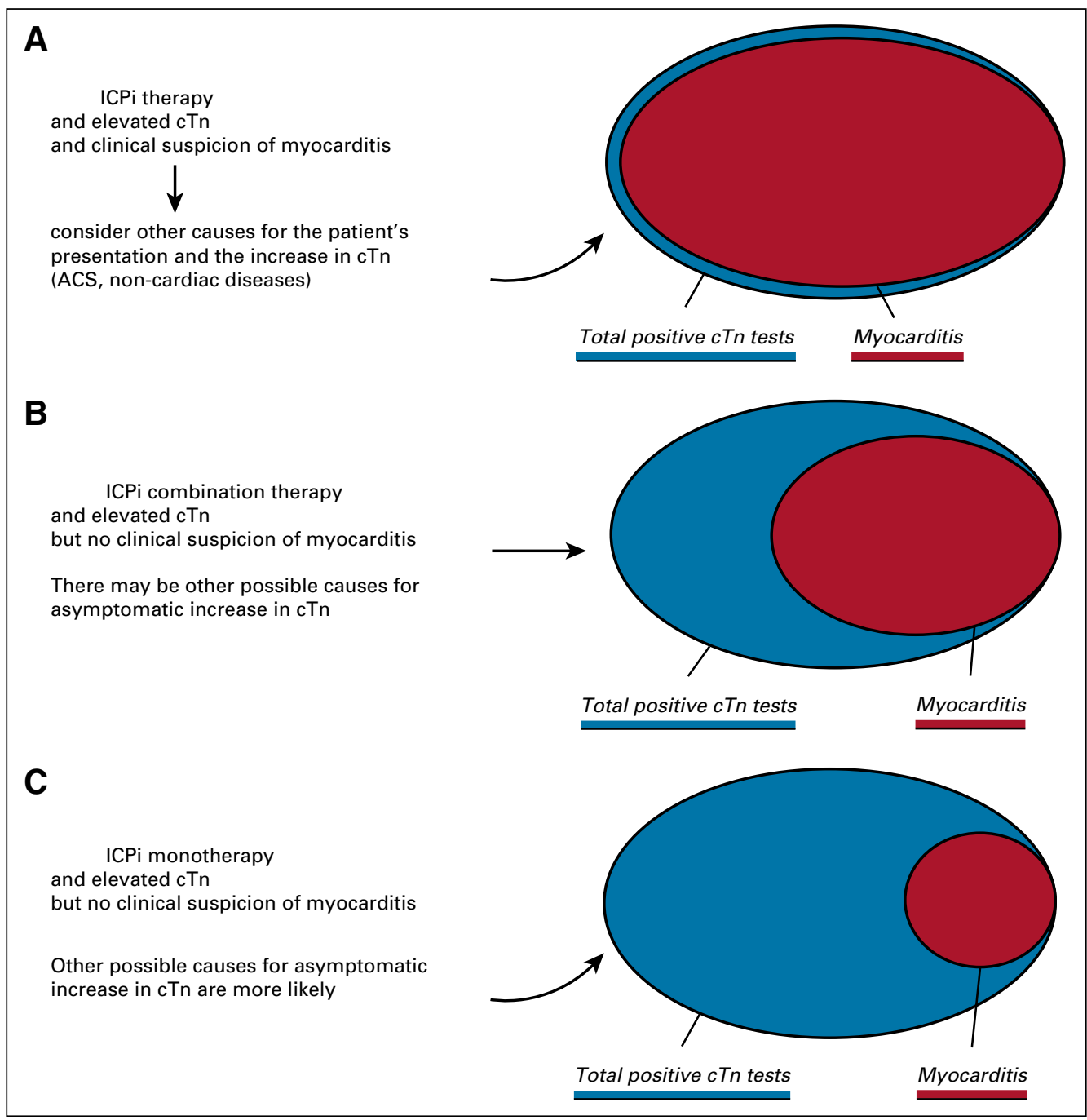

FIG 1. Diverse value of positive cardiac troponin (cTn) testing in patients who receive immune checkpoint inhibitor (ICPi) therapy with various clinical presentations. (A) A clinical scenario suggestive for myocarditis that complicates treatment with ICPis in which cTn is elevated. Especially if other causes of increased cTn are reasonably excluded, such as acute coronary syndrome (ACS) and noncardiac disorders, the high cTn concentration will reflect an ongoing myocarditis in the majority of all tested patients (red and blue circles, respectively). (B) The situation where cTn is assessed in patients who receive combination immune therapy but without any clinical clues of myocarditis. Because dual ICPi treatment is particularly associated with the risk of developing myocarditis, there will be patients with elevated cTn levels who have asymptomatic or atypical myocardial inflammation. However, the increase in cTn may be caused by many other conditions, which would be incorrectly diagnosed as myocarditis on the sole result of cTn testing. Note that even healthy individuals who face cardiac stress (eg, after intense exercise) may have cTn concentrations above the upper reference limit with modern high-sensitivity assays. (C) On-treatment surveillance of patients who receive a single ICPi. The proportion of patients with high cTn and clinically silent or atypical myocarditis will be lower than shown in (B) because the risk of myocarditis with single-drug immune therapy is smaller than with combination treatment. By contrast, other conditions will prevail as the actual cause of cTn elevation. The size of the circles that identify total positive and myocarditis cases is arbitrary. Atypical indicates a myocarditis with uncommon presentation, which may be difficult to recognize even for the specialist (eg, isolated nausea, fever).

ECG alterations, and consistent findings on imaging. Although anecdotal reports suggest that ICPi-related myocarditis may present with isolated abnormal $\mathrm{cTn},{ }^{18}$ this is not what usually happens according to the published experience..$^{2-4}$ This also is not the case with myocarditis in general for which two or more criteria (significant changes in
cTn, ECG, or imaging) are requested for the clinical diagnosis if the patient is asymptomatic. ${ }^{15}$ In the absence of a suggestive clinical picture, myocarditis is a rarer cause of cTn elevation than, for instance, ACS or noncardiac disease, even in patients who receive two ICPis (Fig 1B). This concept has practical implications. Currently, it is advised to hold 
ICPis for any-grade toxicity, including modifications of cardiac biomarkers, and to permanently discontinue them in case of even mild symptoms. ${ }^{16}$ However, because symptoms may be attributed to myocarditis despite being of other origin, a substantial risk of abandoning immunotherapy unnecessarily exists.

Systematic cTn testing also has been suggested during ICPi monotherapy..$^{19}$ Here, the sensitivity of such a screening is expected to be even lower because myocarditis induced by single ICPi treatment is less frequent than by treatment with two ICPis ${ }^{4}$ (Fig 1C). As on-treatment surveillance approach with serial cTn determination, we measured cTnl levels in 59 patients treated with nivolumab for non-small-cell lung cancer, the majority of whom did not have a history of cardiovascular disease, diabetes, or chronic kidney disease..$^{20}$ One patient had a cTnl value above the URL and another six showed an increased concentration during treatment, all without overt cardiovascular events. Yet, only one patient was deemed as possibly having subclinical myocarditis

\section{AFFILIATIONS}

${ }^{1}$ Istituto di Ricovero e Cura a Carattere Scientifico Ospedale Policlinico San Martino, Genoa, Italy

${ }^{2}$ University of Genova, Genoa, Italy

${ }^{3}$ Fondazione Istituto di Ricovero e Cura a Carattere Scientifico Ca' Granda Ospedale Maggiore Policlinico, Milan, Italy

\section{CORRESPONDING AUTHOR}

Pietro Ameri, MD, PhD, Department of Internal Medicine and Center of Excellence for Biomedical Research, University of Genova, Viale Benedetto XV, 6-16132 Genoa, Italy; e-mail: pietroameri@unige.it. upon cardiologic evaluation by the cardio-oncologist, with the others ascribed to myocardial ischemia secondary to a mismatch between oxygen demand and supply. ${ }^{20}$

\section{Conclusion}

Major concerns exist about assessing cTn to detect asymptomatic myocarditis during ICPi treatment. Dedicated prospective studies are needed, with a particular focus on patients at higher risk, such as those prescribed combination immune therapy. ECG is widespread and easily readable, but it is neither sensitive nor specific enough for myocarditis screening. The role of other biomarkers or imaging techniques, if any, is still to be investigated. What should be done then? With the watchful awareness about the possibility of ICPitriggered myocarditis, all diagnostic tests, including cTn, should be used wisely and guided by clinical reasoning. Intense interaction between the oncologist and cardio-oncologist (or cardiologist) is crucial.
AUTHORS' DISCLOSURES OF POTENTIAL CONFLICTS OF INTEREST AND DATA AVAILABILITY STATEMENT

Disclosures provided by the authors and data availability statement (if applicable) are available with this article at DOI https://doi.org/10.1200/ JCO.18.02464

\section{AUTHOR CONTRIBUTIONS}

Manuscript writing: All authors

Final approval of the manuscript: All authors

Accountable for all aspects of the work: All authors

\section{REFERENCES}

1. Spallarossa P, Meliota G, Brunelli C, et al: Potential cardiac risk of immune-checkpoint blockade as anticancer treatment: What we know, what we do not know, and what we can do to prevent adverse effects. Med Res Rev 38:1447-1468, 2018

2. Johnson DB, Balko JM, Compton ML, et al: Fulminant myocarditis with combination immune checkpoint blockade. N Engl J Med 375:1749-1755, 2016

3. Mahmood SS, Fradley MG, Cohen JV, et al: Myocarditis in patients treated with immune checkpoint inhibitors. J Am Coll Cardiol 71:1755-1764, 2018

4. Salem JE, Manouchehri A, Moey M, et al: Cardiovascular toxicities associated with immune checkpoint inhibitors: An observational, retrospective, pharmacovigilance study. Lancet Oncol 19:1579-1589, 2018

5. Neilan TG, Rothenberg ML, Amiri-Kordestani L, et al: Myocarditis associated with immune checkpoint inhibitors: An expert consensus on data gaps and a call to action. Oncologist 23:874-878, 2018

6. Wang DY, Okoye GD, Neilan TG, et al: Cardiovascular toxicities associated with cancer immunotherapies. Curr Cardiol Rep 19:21, 2017

7. Eggers KM, Lindahl B: Application of cardiac troponin in cardiovascular diseases other than acute coronary syndrome. Clin Chem 63:223-235, 2017

8. Thygesen K, Alpert JS, Jaffe AS, et al: Fourth universal definition of myocardial infarction (2018). Eur Heart J 39:3757-3758, 2018

9. Sarkisian L, Saaby L, Poulsen TS, et al: Prognostic impact of myocardial injury related to various cardiac and noncardiac conditions. Am J Med 129:506-514.e1, 2016

10. Jaffe AS, Vasile VC, Milone M, et al: Diseased skeletal muscle: A noncardiac source of increased circulating concentrations of cardiac troponin T. J Am Coll Cardiol 58:1819-1824, 2011

11. Anquetil C, Salem JE, Lebrun-Vignes B, et al: Immune checkpoint inhibitor-associated myositis. Circulation 138:743-745, 2018

12. Ammirati E, Cipriani M, Moro C, et al: Clinical presentation and outcome in a contemporary cohort of patients with acute myocarditis. Circulation 138 : 1088-1099, 2018

13. Mahajan VS, Jarolim P: How to interpret elevated cardiac troponin levels. Circulation 124:2350-2354, 2011

14. de Lemos JA, Drazner MH, Omland T, et al: Association of troponin T detected with a highly sensitive assay and cardiac structure and mortality risk in the general population. JAMA 304:2503-2512, 2010

15. Caforio AL, Pankuweit S, Arbustini E, et al: Current state of knowledge on aetiology, diagnosis, management, and therapy of myocarditis: A position statement of the European Society of Cardiology Working Group on Myocardial and Pericardial Diseases. Eur Heart J 34:2636-2648, 2013

16. Brahmer JR, Lacchetti C, Schneider BJ, et al: Management of immune-related adverse events in patients treated with immune checkpoint inhibitor therapy: American Society of Clinical Oncology clinical practice guideline. J Clin Oncol 36:1714-1768, 2018 
17. Puzanov I, Diab A, Abdallah K, et al: Managing toxicities associated with immune checkpoint inhibitors: Consensus recommendations from the Society for Immunotherapy of Cancer (SITC) Toxicity Management Working Group. J Immunother Cancer 5:95, 2017

18. Norwood TG, Westbrook BC, Johnson DB, et al: Smoldering myocarditis following immune checkpoint blockade. J Immunother Cancer 5:91, 2017

19. Ganatra S, Neilan TG: Immune checkpoint inhibitor-associated myocarditis. Oncologist 23:879-886, 2018

20. Sarocchi M, Grossi F, Arboscello E, et al: Serial troponin for early detection of nivolumab cardiotoxicity in advanced non-small cell lung cancer patients. Oncologist 23:936-942, 2018 


\section{Identification and Management of Immune Checkpoint Inhibitor-Related Myocarditis: Use Troponin Wisely}

The following represents disclosure information provided by authors of this manuscript. All relationships are considered compensated. Relationships are self-held unless noted. I = Immediate Family Member, Inst = My Institution. Relationships may not relate to the subject matter of this manuscript. For more information about ASCO's conflict of interest policy, please refer to www.asco.org/rwc or ascopubs.org/jco/site/ifc.

Paolo Spallarossa

Honoraria: Incyte, TEVA Pharmaceuticals Industries, Daiichi Sankyo, MSD Consulting or Advisory Role: Incyte

Travel, Accommodations, Expenses: Firma

Matteo Sarocchi

Honoraria: Incyte

Travel, Accommodations, Expenses: Bayer AG

Francesco Grossi

Consulting or Advisory Role: MSD, Bristol-Myers Squibb, AstraZeneca, Roche, Pfizer

Speakers' Bureau: MSD, Bristol-Myers Squibb, AstraZeneca, Roche, Pierre Fabre, Amgen, Celgene, Eli Lilly, Pfizer

Research Funding: Bristol-Myers Squibb

Travel, Accommodations, Expenses: Bristol-Myers Squibb, MSD, Roche, AstraZeneca, Pierre Fabre, Celgene, Amgen, Eli Lilly, Novartis
Paola Queirolo

Consulting or Advisory Role: Roche, Genentech, Novartis, MSD, Bristol-Myers Squibb, Pierre Fabre, Sanofi

Gabriele Zoppoli

Travel, Accommodations, Expenses: Novartis, Roche, Pfizer, Eli Lilly, Amgen

Pietro Ameri

Honoraria: Daiichi Sankyo

Consulting or Advisory Role: Boehringer Ingelheim, Daiichi Sankyo, Boehringer Ingelheim, Novartis, GlaxoSmithKline, Bayer AG

No other potential conflicts of interest were reported. 